Article

\title{
Transition Metal Dichalcogenides as Strategy for High Temperature Electron-Hole Superfluidity
}

\author{
Sara Conti ${ }^{1,2, * \mathbb{C}}$, David Neilson ${ }^{2}$, François M. Peeters ${ }^{2}{ }^{\mathbb{D}}$ and Andrea Perali ${ }^{3}$ \\ 1 Physics Division, School of Science \& Technology, Università di Camerino, 62032 Camerino (MC), Italy \\ 2 Department of Physics, University of Antwerp, Groenenborgerlaan 171, B-2020 Antwerpen, Belgium; \\ dneilson@ftml.net (D.N.); francois.peeters@uantwerpen.be (F.M.P.) \\ 3 Supernano Laboratory, School of Pharmacy, Università di Camerino, 62032 Camerino (MC), Italy; \\ andrea.perali@unicam.it \\ * Correspondence: sara.conti@unicam.it
}

Received: 21 February 2020; Accepted: 20 March 2020; Published: 22 March 2020

\begin{abstract}
Condensation of spatially indirect excitons, with the electrons and holes confined in two separate layers, has recently been observed in two different double layer heterostructures. High transition temperatures were reported in a double Transition Metal Dichalcogenide (TMD) monolayer system. We briefly review electron-hole double layer systems that have been proposed as candidates for this interesting phenomenon. We investigate the double TMD system $\mathrm{WSe}_{2} / \mathrm{hBN} / \mathrm{MoSe}_{2}$, using a mean-field approach that includes multiband effects due to the spin-orbit coupling and self-consistent screening of the electron-hole Coulomb interaction. We demonstrate that the transition temperature observed in the double TMD monolayers, which is remarkably high relative to the other systems, is the result of (i) the large electron and hole effective masses in TMDs, (ii) the large TMD band gaps, and (iii) the presence of multiple superfluid condensates in the TMD system. The net effect is that the superfluidity is strong across a wide range of densities, which leads to high transition temperatures that extend as high as $T_{B K T}=150 \mathrm{~K}$.
\end{abstract}

Keywords: superfluidity; exciton; electron-hole; transition-metal-dichalcogenides; multibandsuperconductivity; multicomponent-superfluidity

\section{Introduction}

Spatially indirect excitons are states of electrons and holes bound by their Coulomb attraction. The electrons and holes are confined to two separated quantum wells or quasi two-dimensional (2D) layers, placed very close to each other. There is a great deal of interest in spatially indirect excitons condensing into a quantum coherent state.

Electron-hole superfluidity was first proposed for a conventional semiconductor heterostructure of electron and hole doped Double Quantum-Wells (DQW) [1-3]. In GaAs, the quantum wells are separated by a thin insulating barrier of $\mathrm{Al}_{x} \mathrm{Ga}_{1-x}$ As which blocks recombination of the electrons and holes [4]. The strength of the electron-hole pairing is primarily controlled by the average effective separation between the electrons and the holes, determined by the thickness of the insulating barrier and by the widths of the quantum wells. It is challenging to identify the optimal configurations for the GaAs wells and the insulating barriers [5]. The wells cannot be too narrow or else interface roughness scattering, arising from insulating barrier $\mathrm{Al}$ atoms diffusing into the well regions, makes mobility impractically low. At 
high densities, when the system approaches the weakly interacting regime, the competition between intralayer and interlayer correlations becomes strong, and the screening of the electron-hole interaction is predicted to kill the superfluidity [6]. Since the average effective separation is large, there is superfluidity only for densities $\lesssim 10^{10} \mathrm{~cm}^{-2}$. The predicted transition temperatures for this system are relatively low, $T_{B K T} \sim 1 \mathrm{~K}$. To date, there have been no definitive observations of superfluidity in GaAs DQWs [5]. It would be particularly interesting to observe superfluidity in this system, because it is likely to have a rich phase diagram of exotic superfluid phases [7] due to the large difference in electron and hole effective masses in GaAs.

The discovery of graphene [8] opened the way to atomically-thin materials which bypass the structural challenges of the GaAs DQWs. Double Monolayer Graphene (DMG), consisting of two monolayers of graphene separated by only $\sim 1 \mathrm{~nm}$ of hexagonal Boron Nitride (hBN) as the insulating barrier [9], was proposed [10,11] and investigated [12]. However the linear dispersion of the energy bands which is almost unique to monolayer graphene, proved to be an enormous obstacle for accessing the strongly interacting regime where superfluidity would occur. In the weakly interacting regime, screening kills the superfluidity [13].

Double Bilayer Graphene (DBG), using bilayer graphene in place of monolayer graphene, was proposed to overcome this problem [14]. Unlike monolayer graphene, the energy dispersion in bilayer graphene is approximately parabolic, as in conventional semiconductors. A recent experiment confirmed the prediction in Reference [14]: enhanced tunneling conductance was reported, which is a signature of electron-hole superfluidity, in a DBG system with a $1.4 \mathrm{~nm} \mathrm{WSe}{ }_{2}$ insulating barrier [15]. This signature was observed only at lower densities and is in quantitative agreement with the theoretical predictions [16] of an upper limit of the carrier density for the superfluidity. Above this threshold density, screening kills the superfluidity. The observed transition temperature is low, $T_{\mathcal{C}} \sim 1.5 \mathrm{~K}$. Reference [14] had predicted a maximum transition temperature of $17 \mathrm{~K}$ in a DBG with a $1.4 \mathrm{~nm}$ hBN barrier. Reference [16] pointed out the importance of the strong interband screening from the valence band, a large effect here because of the very small band gap in bilayer graphene [17]. The effect of this additional screening is to reduce the threshold density and the maximum transition temperature.

The development of other 2D materials like the Transition Metal Dichalcogenides (TMDs) followed graphene. The TMD monolayers $\mathrm{MoS}_{2}, \mathrm{MoSe}_{2}, \mathrm{WS}_{2}$, and $\mathrm{WSe}_{2}$ are semiconductors with direct band gaps comparable to that in GaAs [18,19]. The large band gap eliminates the detrimental effects of the interband screening mentioned above for graphene. The electron and hole effective masses in TMDs are larger than for bilayer graphene, and this further increases the coupling strength of the electron-hole pairs [20]. Reference [21] proposed the TMD heterostructure $\mathrm{MoSe}_{2}-\mathrm{hBN}-\mathrm{WSe} \mathrm{e}_{2}$ to observe electron-hole superfluidity and to investigate additional novel multicomponent effects resulting from the strong spin-orbit coupling. The splitting of the valence bands $\lambda_{v}$ is an order of magnitude larger than the splitting of the conduction bands $\lambda_{c}$. The resulting misalignment of the electron and hole bands fundamentally changes the multicomponent nature of the superfluidity. The authors already predicted maximum transition temperatures as high as $T_{B K T} \sim 100 \mathrm{~K}$ [21], and very recently enhanced tunneling conductance signatures of Bose-Einstein Condensation (BEC) was reported in this same system with transition temperature $T_{\mathcal{C}} \sim 100 \mathrm{~K}$ [22] consistent with these predictions.

Table 1 collects the relevant properties and results for the double layer systems proposed for electron-hole superfluidity. 
Table 1. Experimental (E) and theoretical (T) properties and results of the different systems. $m_{e}^{*}$ and $m_{h}^{*}$ : electron and hole effective masses; $E_{g}$ : energy band gap; $d$ : layer separation; $n_{0}$ : superfluid threshold density $\left(10^{11} \mathrm{~cm}^{-2}\right) ; T_{c}$ : transition temperature for superfluidity.

\begin{tabular}{cccccccccc}
\hline & $m_{e}^{*}\left(m_{e}\right)$ & $m_{h}^{*}\left(m_{e}\right)$ & $E_{g}(\mathbf{e V})$ & $d^{T}(\mathbf{n m})$ & $d^{E}(\mathbf{n m})$ & $n_{0}^{T}$ & $n_{0}^{E}$ & $T_{c}^{T}(\mathbf{K})$ & $T_{c}^{E}(\mathbf{K})$ \\
\hline DQW & 0.067 & 0.3 & 1.5 & $15[5]$ & $25[2,3]$ & $<0.7[5]$ & - & $\sim 1[5]$ & - \\
DMG & 0 & 0 & 0 & $1.0[11]$ & $1.0[12]$ & - & - & - & - \\
DBG & 0.04 & 0.04 & $\leq 0.25$ & $1.4[14,16]$ & $1.4[15]$ & $7.0[16]$ & $8.0[15]$ & $17[14]$ & $1.5[15]$ \\
TMD & $0.3-0.5$ & $0.4-0.6$ & $1.5-2.0$ & $1.0[21]$ & $1.0[22]$ & $150[21]$ & $10[22]$ & $\gtrsim 100[21]$ & $100[22]$ \\
\hline
\end{tabular}

\section{Results}

In this paper we investigate the effects of multicomponent superfluidity in double TMD monolayers, and show that these effects contribute to the high transition temperatures observed in a TMD system. We present results for the specific system $\mathrm{WSe}_{2} / \mathrm{hBN} / \mathrm{MoSe}_{2}$, with $n$-doped $\mathrm{WSe}_{2}$ and $p$-doped $\mathrm{MoSe}_{2}$, and then draw general conclusions for the class of semiconductor TMDs. The splitting of the conduction and valence bands by spin-orbit coupling into multibands (Figure 1) consists of two spin-polarized subbands. The subbands are concentric and this makes the superfluidity resemble multiband superconductivity in certain aspects.

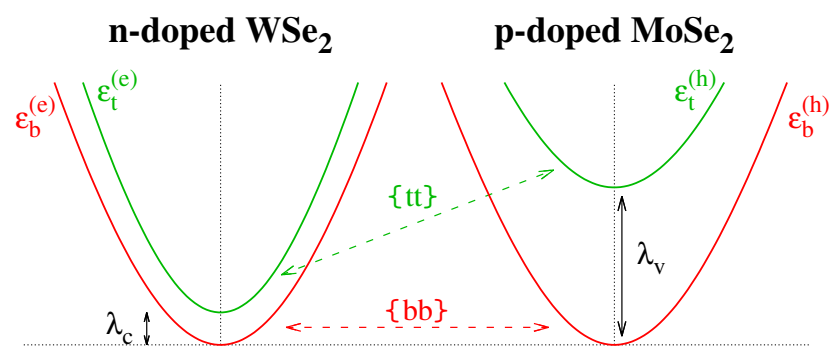

Figure 1. Low-energy multiband structure resulting from spin-orbit coupling in $n-\mathrm{WSe} / \mathrm{hBN} / \mathrm{MoSe}_{2}-p$. The $\mathrm{MoSe}_{2}$ bands are the valence bands after a standard particle-hole transformation to positive energies. Because of the large band gap in TMDs, we are able to neglect the remote bands. $\lambda_{c}\left(\lambda_{v}\right)$ is the spin-orbit splitting of the conduction (valence) bands. The dashed arrows labelled $\{b b\}$ and $\{t t\}$ indicate schematically the pairing channels.

We explain in the Methods section that there are only two active pairing channels in this system. In Figure 1, we label these $\{b b\}$ and $\{t t\}$ for pairing of carriers from the bottom-bottom and top-top bands. These channels are associated with two condensates with different superfluid gaps $\Delta_{b b}$ and $\Delta_{t t}$, given by Equations (8) and (9), that depend on the number of pairs in the respective bands.

Figure 2a shows the maximum of the superfluid gaps $\Delta_{b b}$ and $\Delta_{t t}$ as a function of the carrier density $n$. Figure $2 \mathrm{~b}$ shows the corresponding evolution of the chemical potential $\mu=\frac{1}{2}\left(\mu^{(e)}+\mu^{(h)}\right)$. Because of the interactions, $\mu$ is only equal to the Fermi energy in the weakly interacting regime at high densities [23].

To identify the strongly and weakly interacting regions, we use the condensate fraction $C$. This is defined as the fraction of electron-hole pairs relative to the total number of electrons or holes (see Equation (17)). Figure 3 shows the condensate fractions $C_{b b}$ and $C_{t t}$ as functions of density. For $C>0.8$ the condensate is said to be in the strong-coupled BEC regime, for $0.2 \leq C \leq 0.8$ in the crossover regime, and for $C<0.2$ in the weak-coupled regime. 

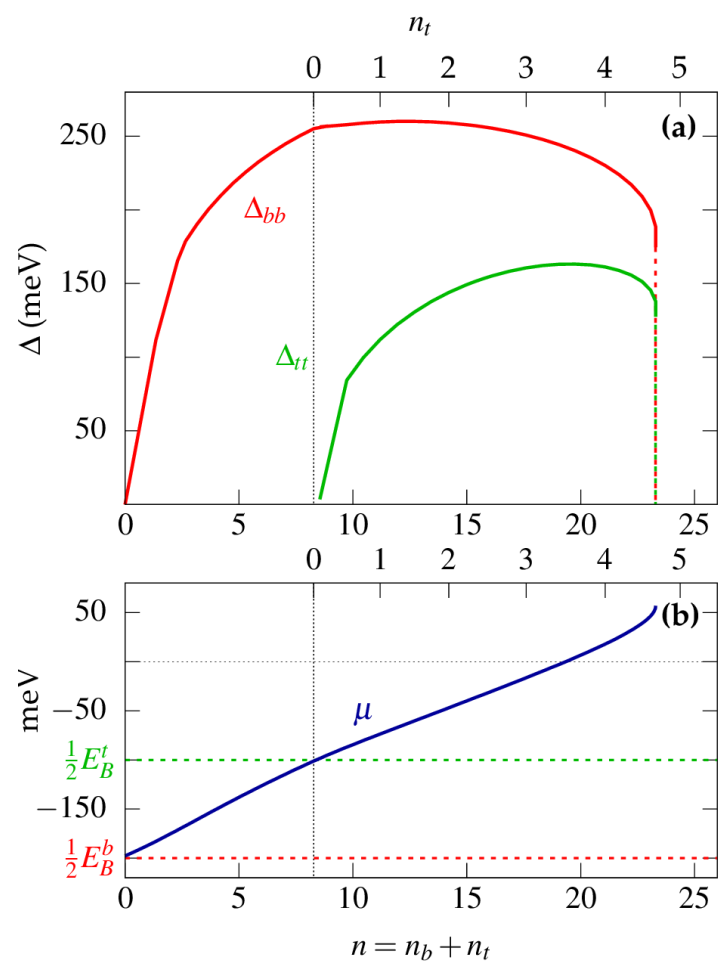

Figure 2. (a) The maximum of the superfluid gaps $\Delta_{b b}$ and $\Delta_{t t}$ as a function of the density $n$. For reference, the upper horizontal axis shows the density of the top bands $n_{t}$. Both densities have units of $10^{12} \mathrm{~cm}^{-2}$. (b) Chemical potential as a function of the density $n$. The zero energy is fixed at the minimum of the bottom bands $\varepsilon_{b}(0)$. The $E_{B}^{b} / 2$ and $E_{B}^{t} / 2$ are the two-body bound state energies.

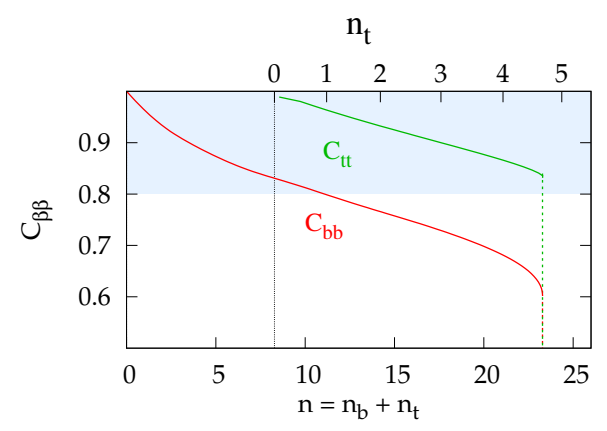

Figure 3. Condensate fraction $C_{b b}$ and $C_{t t}$ as a function of $n\left(10^{12} \mathrm{~cm}^{-2}\right)$. The upper horizontal axis shows the density of the top bands $n_{t}\left(10^{12} \mathrm{~cm}^{-2}\right)$. The blue shaded area is the BEC regime

At low densities, electrons and holes occupy only the bottom bands so there is only a $\{b b\}$ condensate. In the low density limit, the paired electrons and holes occupy the two-body bound state with its binding energy $E_{B}^{b} \sim 375 \mathrm{meV}$. The chemical potential for this state is given by the one-band limit, $\mu \sim-E_{B}^{b} / 2$, (Figure $2 b)[24,25]$. In this case, the $\{b b\}$ condensate is in the BEC regime, with condensate fraction $C_{b b}>0.8$. 
As the density is increased $\Delta_{b b}$ also increases, since the number of electrons and holes available for pairing increases. The pairs start to overlap, and this results in an increasing chemical potential $\mu$ and a decreasing $C_{b b}$. When $C_{b b}$ drops below 0.8 , the $\{b b\}$ condensate enters the crossover regime.

The misalignment between the top electron and top hole bands is $\delta \lambda=\lambda_{v}-\lambda_{c}$ (Figure 1). Thus in the low density limit of the top bands, the paired electrons and holes will occupy a two-body bound state with a binding energy that lies $E_{B}^{b}-\delta \lambda$ below the top band $\varepsilon_{t}^{(e)}$. Relative to the zero of energy, the two-body bound state for the top bands is located at

$$
-\frac{1}{2} E_{B}^{t}=-\frac{1}{2}\left(E_{B}^{b}-\delta \lambda\right)+\lambda_{c} .
$$

When $\mu$ moves above $-\frac{1}{2} E_{B}^{t}$, the $\{t t\}$ condensate can start to be populated and $\Delta_{t t}$ becomes non-zero. The superfluidity may be one-component at low density and two-component at higher densities. A similar behaviour has been observed in multiband superconductivity [26]. We recall that in both systems the multibands are concentric. In contrast with the $\{b b\}$ condensate, the $\{t t\}$ condensate is always in the BEC regime $\left(C_{t t}>0.8\right.$ in Figure 3). This results in an interesting new mixture of the $\{b b\}$ and $\{t t\}$ condensates that are in different regimes of pairing.

With increasing density, $\Delta_{b b}$ passes through a maximum and then begins to decrease. This is an effect of screening which becomes increasingly important and weakens the interaction. $\Delta_{t t}$ follows a similar sequence of behaviour. The step function in Equation (9), reduces the pairing strength because of the misalignment of the top bands. This is the reason that $\Delta_{t t}$ is everywhere weaker than $\Delta_{b b}$.

The weakening due to screening is accelerated due to the build up of the free carriers in the bottom bands. Eventually screening kills the superfluidity, $\Delta$ drops sharply to zero in both bottom and top channels at the same threshold density $n_{0}$ [14]. We use the threshold density to determine the maximum transition temperature for superfluidity $T_{B K T}$ (See Equation (18)). We find $T_{B K T}$ can be as high as $120 \mathrm{~K}$ in this system.

The two-body bound state can only exist if it lies below the minimum of the top bands. Otherwise there is a decay channel to continuum states. This means that in Equation (1), $\left(E_{B}^{b}-\delta \lambda\right)$ must be positive for a two-body bound state in the top bands to exist. This is a condition for the second condensate to exist: $\delta \lambda<E_{B}^{b}$. Table 2a shows $\left(E_{B}^{b}-\delta \lambda\right)$ for different combinations of TMD monolayers. Table $2 \mathrm{~b}$ gives the corresponding two-body binding energy for the $\{b b\}$ bands, $E_{B}^{b}$, for different combinations of TMD monolayers.

Table 2. (a) $\left(E_{B}^{b}-\delta \lambda\right)$ in meV for different combinations of TMD monolayers. The $E_{B}^{b}$ are calculated for double TMD monolayers with separation $d=1 \mathrm{~nm} . \delta \lambda$ is determined from Table 3. Multicomponent superfluidity is possible only when $\left(E_{B}^{b}-\delta \lambda\right)>0$ (marked in bold). (b) Corresponding two-body binding energy for the $\{b b\}$ bands: $E_{B}^{b}$ in meV.

\begin{tabular}{cccccccccc}
\hline (a) & p-MoS & p-MoSe & p-WS & p-WSe & (b) & p-MoS & p-MoSe & p-WS & p-WSe \\
\hline n-MoS $_{\mathbf{2}}$ & $\mathbf{2 4 9}$ & $\mathbf{2 2 8}$ & -37 & -69 & n-MoS & 396 & 405 & 390 & 388 \\
n-MoSe $_{2}$ & $\mathbf{2 7 6}$ & $\mathbf{2 5 3}$ & -9 & -49 & n-MoSe & 405 & 412 & 400 & 390 \\
n-WS $_{\mathbf{2}}$ & $\mathbf{2 5 2}$ & $\mathbf{2 3 2}$ & -28 & -65 & n-WS & 375 & 385 & 375 & 368 \\
n-WSe $_{\mathbf{2}}$ & $\mathbf{2 6 0}$ & $\mathbf{2 3 3}$ & -24 & -57 & n-WSe & 372 & 375 & 368 & 365 \\
\hline
\end{tabular}

\section{Discussion}

The strikingly high transition temperatures for electron-hole superfluidity in double TMD monolayer system comes from two properties in which they greatly differ from other double layer systems: (i) high threshold densities for superfluidity and (ii) multicomponent superfluidity that can be switched on and off. 
(i) In 2D, the superfluid transition temperatures are given by $T_{B K T}$, the Berezinskii-Kosterlitz-Thouless (BKT) temperature [27]. $T_{B K T}$ is proportional to the density (see Equation (18)), so to achieve high transition temperature the density must be maximized. The threshold densities $n_{0}$ in Figure 2a are much larger than $n_{0} \sim 8 \times 10^{11} \mathrm{~cm}^{-2}$ observed in DBG [15,16], $n_{0} \sim 4 \times 10^{12} \mathrm{~cm}^{-2}$ for phosphorene [28], and $n_{0} \sim 5 \times 10^{10}$ $\mathrm{cm}^{-2}$ for GaAs [5]. The reason is the large electron and hole effective masses in the TMDs [20] which lead to strong binding energies and thus large superfluid gaps $\Delta$.

(ii) While at first sight, the coupling in DBG between the conduction and valence band condensates should reinforce the superfluid gaps [29], because of the small band gaps in bilayer graphene there are a large number of free carriers that are easily excited from the valence band, and these greatly enhance the screening [16]. The enhanced screening weakens the superfluid gaps. Reference [16] found that this effect dominate over reinforcement of the gaps.

In TMDs, the large band gap means that screening from the remote bands is negligible. Because of spin selection, the condensates are not directly coupled in the gap equations (Equations (8) and (9)). In this case there is a great advantage in having a second condensate for the following reason. The pairing in the $\{t t\}$ condensate removes free carriers in the top bands that would otherwise strengthen the screening. Thus two-component superfluidity persists up to higher densities compared to the one-component system.

To identify the double TMD monolayers with the highest transition temperatures we look for the highest binding energies in combination with multicomponent superfluidity. Of the double monolayers which are predicted in Table $2 \mathrm{a}$ to exhibit multicomponent superfluidity (marked in bold), we see from Table $2 b$, it is $\mathrm{MoSe}_{2}-\mathrm{MoSe}_{2}$ which has the highest two-body binding energy. Hence $\mathrm{MoSe}_{2}-\mathrm{MoSe}_{2}$ has the largest transition temperature: $T_{B K T} \sim 160 \mathrm{~K}$.

\section{Materials and Methods}

\subsection{Materials}

We are focusing attention on the Transition Metal Dichalcogenides monolayers $\mathrm{MoS}_{2}, \mathrm{MoSe}_{2}, \mathrm{WS}_{2}$, and $\mathrm{WSe}_{2}$, semiconductors with large and direct band gaps, with low-lying nearly parabolic bands and strong spin-orbit coupling. Table 3 gives the parameters for these TMDs monolayers.

Table 3. TMD monolayer effective masses, band gap $\left(E_{g}\right)$, and spin-orbit coupling splitting of conduction bands $\left(\lambda_{c}\right)$ and valence bands $\left(\lambda_{v}\right)[30-32]$.

\begin{tabular}{cccccc}
\hline & $m_{e}^{*}\left(m_{e}\right)$ & $m_{h}^{*}\left(m_{e}\right)$ & $E_{g}(\mathrm{eV})$ & $\lambda_{c}(\mathrm{eV})$ & $\lambda_{v}(\mathrm{eV})$ \\
\hline $\mathbf{M o S}_{2}$ & 0.40 & 0.48 & 1.66 & -0.003 & 0.15 \\
$\mathbf{M o S e}_{2}$ & 0.43 & 0.50 & 1.47 & -0.021 & 0.18 \\
$\mathbf{W S}_{2}$ & 0.33 & 0.30 & 1.79 & 0.027 & 0.43 \\
$\mathbf{W S e}_{2}$ & 0.36 & 0.30 & 1.60 & 0.038 & 0.46 \\
\hline
\end{tabular}

The electronic dispersion in a single valley is [33]:

$$
\varepsilon_{\sigma, \pm}(k)=\sigma \frac{\lambda_{c}+\lambda_{v}}{2} \pm \sqrt{a^{2} t^{2} k^{2}+\frac{\left(E_{g}-\sigma\left(\lambda_{v}-\lambda_{c}\right)\right)^{2}}{4}},
$$

with the plus (minus) sign labelling the conduction (valence) band and $\sigma= \pm$ as spin index.

We focus on the TMD heterostructure WSe $2 / \mathrm{hBN} / \mathrm{MoSe}_{2}$, with the WSe 2 -doped and the $\mathrm{MoSe}_{2}$ p-doped. The monolayers are separated by an insulating trilayer of hexagonal Boron Nitride (hBN) with thickness $d=1 \mathrm{~nm}$ and dielectric constant $\epsilon=2$ [34]. The barrier inhibits electron-hole recombination [9], and avoids hybridization between the bands $[35,36]$ 


\subsection{Method}

Because of the large band gaps, we need only to consider conduction band processes [16,29] (see Figure 1). For each of the monolayers, we label the bottom and top conduction subbands $\beta=b$ and $\beta=t$. Due to the large valley separation in momentum space, inter-valley scattering is negligible, so the effect of the two valleys appears only in a valley degeneracy factor, $g_{v}=2$.

The multiband electron-hole Hamiltonian is,

$$
H=\sum_{k, \beta}\left\{\xi_{\beta}^{(e)}(k) c_{\beta, k}^{\dagger} c_{\beta, k}+\xi_{\beta}^{(h)}(k) d_{\beta, k}^{+} d_{\beta, k}\right\}+\sum_{\substack{k, k^{\prime}, q \\ \beta, \beta^{\prime}}} V_{k k^{\prime}}^{D} c_{\beta, k+q / 2}^{+} d_{\beta,-k+q / 2}^{+} c_{\beta^{\prime}, k^{\prime}+q / 2} d_{\beta^{\prime},-k^{\prime}+q / 2} .
$$

For the $n$-doped monolayer, $c_{\beta, k}^{\dagger}$ and $c_{\beta, k}$ are the creation and annihilation operators for electrons in conduction subband $\beta$. For the $p$-doped monolayer, $d_{\beta, k}^{\dagger}$ and $d_{\beta, k}$ are the corresponding operators for holes. The kinetic energy terms are $\xi_{\beta}^{(i)}(k)=\varepsilon_{\beta}^{(i)}(k)-\mu^{(i)}$ where $\varepsilon_{\beta}^{(i)}(k)$ is the energy dispersion for the $i=e, h$ monolayer, as in Equation (2). We consider only equal carrier densities $n^{e}=n^{h}=n$, so the chemical potentials $\mu=\frac{1}{2}\left(\mu^{(e)}+\mu^{(h)}\right) \cdot V_{k k^{\prime}}^{D}$ is the bare attractive Coulomb interaction between electrons and holes in the opposite monolayers,

$$
V_{k k^{\prime}}^{D}=-V_{k k^{\prime}}^{S} e^{-d\left|\mathbf{k}-\mathbf{k}^{\prime}\right|}, \quad V_{k k^{\prime}}^{S}=\frac{2 \pi e^{2}}{\epsilon} \frac{1}{\left|\mathbf{k}-\mathbf{k}^{\prime}\right|},
$$

where $d$ is the thickness of the barrier. $V_{k k^{\prime}}^{S}$ is the bare repulsive Coulomb interaction between carriers in the same monolayer.

In contrast with conventional BCS pairing, the Coulomb pairing interaction is independent of the electron and hole spins. There are four possible electron-hole pairings, corresponding to four superfluid condensates $[37,38]$. We introduce the temperature dependent normal and anomalous multiband Matsubara Green functions, with subband indices $\alpha$ and $\beta$,

$$
\left\{\begin{array}{l}
\mathcal{G}^{\alpha \beta}(k, \tau)=-<T_{\tau} c_{k}^{\alpha}(\tau) c_{k}^{\beta \dagger}(0)> \\
\mathcal{F}^{\alpha \beta}(k, \tau)=-<T_{\tau} c_{k}^{\alpha}(\tau) d_{k}^{\beta}(0)>
\end{array}\right.
$$

where $T_{\tau}$ is the time-ordering operator. These result in mean field equations for the gaps and the densities [37,38]:

$$
\begin{gathered}
\Delta_{\alpha \beta}(k)=-\frac{T}{L^{2}} \sum_{\substack{\alpha^{\prime}, \beta^{\prime}, k^{\prime}, i \omega_{n}}} F_{k k^{\prime}}^{\alpha \beta \alpha^{\prime} \beta^{\prime}} V_{k k^{\prime}}^{e h} \mathcal{F}^{\alpha^{\prime} \beta^{\prime}}\left(k^{\prime}, i \omega_{n}\right) \\
n_{\alpha \beta}=g_{s} g_{v} \frac{T}{L^{2}} \sum_{k, i \omega_{n}} \mathcal{G}^{\alpha \beta}\left(k, i \omega_{n}\right),
\end{gathered}
$$

where $F_{k k^{\prime}}^{\alpha \beta \alpha^{\prime} \beta^{\prime}}=\left\langle\alpha^{\prime} k^{\prime} \mid \alpha k\right\rangle\left\langle\beta k \mid \beta^{\prime} k^{\prime}\right\rangle$ is the form factor that represents the overlap of the single particle wave functions [39]. Because of the spin polarization in the bands, there is no spin degeneracy, $g_{s}=1$.

We determined that the $\{b t\}$ and $\{t b\}$ cross-pairing terms are negligible. Thus we can focus on intraband pairing, retaining the Green functions and form factors only with $\alpha=\beta\left(\alpha^{\prime}=\beta^{\prime}\right)$. Since the Coulomb interaction $V_{k k^{\prime}}^{e h}$ conserves the spin of the electron-hole pair, there are no spin-flip scattering 
processes. This implies that $F_{k k^{\prime}}^{\beta \beta \beta^{\prime} \beta^{\prime}}=0$ for $\beta \neq \beta^{\prime}$, so there is no Josephson-like pair transfer between the bands [29].

At zero temperature the gap equations are decoupled,

$$
\begin{aligned}
\Delta_{b b}(k) & =-\frac{1}{L^{2}} \sum_{k^{\prime}} F_{k k^{\prime}}^{b b} V_{k k^{\prime}}^{e h} \frac{\Delta_{b b}\left(k^{\prime}\right)}{2 E_{b}\left(k^{\prime}\right)}, \\
\Delta_{t t}(k) & =-\frac{1}{L^{2}} \sum_{k^{\prime}} F_{k k^{\prime}}^{t t} V_{k k^{\prime}}^{e h} \frac{\Delta_{t t}\left(k^{\prime}\right)}{2 E_{t}\left(k^{\prime}\right)} \theta\left[E_{t}^{-}\left(k^{\prime}\right)\right],
\end{aligned}
$$

where we abbreviate the notation $F_{k k^{\prime}}^{\beta \beta \beta^{\prime} \beta^{\prime}} \equiv F_{k k^{\prime}}^{\beta \beta^{\prime}} . E_{\beta}(k)=\sqrt{\xi_{\beta}(k)^{2}+\Delta_{\beta \beta}^{2}(k)}$ is the quasi-particle excitation energy for subband $\beta$, with $\xi_{\beta}(k)=\left(\xi_{\beta}^{(e)}+\xi_{\beta}^{(h)}\right) / 2 . \theta\left[E_{t}^{-}(k)\right]=1-f\left[E_{t}^{-}(k), 0\right]$ is a step function, with $f\left[E_{t}^{-}(k), 0\right]$ the zero temperature Fermi-Dirac distribution. $E_{t}^{ \pm}(k)=E_{t}(k) \pm \delta \lambda / 2$, with $\delta \lambda=\left|\lambda_{v}\right|-\left|\lambda_{c}\right|$ the energy misalignment of the top bands.

Equation (8) has the same form as for a decoupled one-band system, because the two bottom bands are aligned [40]. In contrast, Equation (9) contains the effect of misalignment of the top bands through the term $\theta\left[E_{t}^{-}\left(k^{\prime}\right)\right] \equiv \theta\left[\sqrt{\xi_{t}(k)^{2}+\Delta_{t t}^{2}(k)}-\delta \lambda / 2\right]$. This term drops below unity only at higher densities where the pair coupling strength is weak compared with the misalignment.

$V_{k k^{\prime}}^{e h}$ in Equations (8) and (9) is the screened electron-hole interaction. We use the linear-response Random Phase Approximation for static screening in the superfluid state [16],

$$
V_{k k^{\prime}}^{e h}=\frac{V_{k k^{\prime}}^{D}+\Pi_{a}(q)\left[\left(V_{k k^{\prime}}^{S}\right)^{2}-\left(V_{k k^{\prime}}^{D}\right)^{2}\right]}{1-2\left[V_{k k^{\prime}}^{S} \Pi_{n}(q)+V_{k k^{\prime}}^{D} \Pi_{a}(q)\right]+\left[\Pi_{n}^{2}(q)-\Pi_{a}^{2}(q)\right]\left[\left(V_{k k^{\prime}}^{S}\right)^{2}-\left(V_{k k^{\prime}}^{D}\right)^{2}\right]},
$$

with $q=\left|\mathbf{k}-\mathbf{k}^{\prime}\right| . \Pi_{n}(q)$ is the normal static polarizability in the superfluid state and $\Pi_{a}(q)$ is the anomalous static polarizability [13,14]. The polarizabilties are obtained as loops consisting of two normal or two anomalous Green functions:

$$
\begin{aligned}
& \Pi_{n}(q)=T \frac{g_{s} g_{v}}{L^{2}} \sum_{\beta, k^{\prime}, i \omega_{n}} F_{k k^{\prime}}^{\beta \beta} \mathcal{G}^{\beta \beta}\left(k^{\prime}, i \omega_{n}\right) \mathcal{G}^{\beta \beta}\left(k, i \omega_{n}\right), \\
& \Pi_{a}(q)=T \frac{g_{s} g_{v}}{L^{2}} \sum_{\beta, k^{\prime}, i \omega_{n}} F_{k k^{\prime}}^{\beta \beta} \mathcal{F}^{\beta \beta}\left(k^{\prime}, i \omega_{n}\right) \mathcal{F}^{\beta \beta}\left(k, i \omega_{n}\right) .
\end{aligned}
$$

The sum over the Matsubara frequencies, $\omega_{n}$, is performed at zero temperature.

$\Pi_{n}(q)$ depends on the population of free carriers, while $\Pi_{a}(q)$, with opposite sign, depends on the population of electron-hole pairs. $\Pi_{a}(q)$ is only non-zero in the superfluid state. There is a competition between of $\Pi_{n}(q)$ and $\Pi_{a}(q)$ which is connected to the competition between the populations of the condensed pairs and the free carriers. Screening is associated with the population of charged free carriers. A system with a large superfluid condensate fraction of strong-coupled pairs thus has very weak screening [6].

For a given chemical potential $\mu$, the carrier density $n$ of one monolayer is the sum of the subband carrier densities $n_{b}$ and $n_{t}$, 


$$
\begin{aligned}
n & =g_{s} g_{v} \sum_{\beta=b, t} n_{\beta}, \\
n_{b} & =\frac{1}{L^{2}} \sum_{k} v_{b}^{2}(k), \\
n_{t} & =\frac{1}{L^{2}} \sum_{k}\left[v_{t}^{2}(k) \theta\left[E_{t}^{+}(k)\right]+u_{t}^{2}(k)\left(1-\theta\left[E_{t}^{-}(k)\right]\right)\right],
\end{aligned}
$$

where

$$
v_{\beta}^{2}(k)=\frac{1}{2}\left(1-\frac{\xi_{\beta}(k)}{E_{\beta}(k)}\right) ; \quad u_{\beta}^{2}(k)=\frac{1}{2}\left(1+\frac{\xi_{\beta}(k)}{E_{\beta}(k)}\right),
$$

are the Bogoliubov amplitudes.

The $\Delta_{\beta \beta}$ and $\mu$ shown in Figure 2 are determined by self consistently solving Equations (8) and (9) coupled with Equations (14) and (15).

The condensate fractions $C_{\beta \beta}$ used in Figure 3 to identify the weak- and strong-coupled regimes of the superfluid, are given by $[41,42]$,

$$
C_{\beta \beta}=\frac{\sum_{k} u_{\beta}^{2}(k) v_{\beta}^{2}(k)}{\sum_{k} v_{\beta}^{2}(k)} .
$$

The Berezinskii-Kosterlitz-Thouless transition temperature $T_{B K T}$ [27] for a 2D system with parabolic bands, is determined from $[43,44]$,

$$
T_{B K T}=\frac{\pi}{2} \rho_{s}\left(T_{B K T}\right) \simeq n \frac{\pi \hbar^{2}}{2 g_{s} g_{v} m^{*}} .
$$

$\rho_{s}(T)$ is the superfluid stiffness.

We note that unlike DBG, there are no chiral symmetry degrees of freedom in TMDs. Reference [39] has suggested that these additional degrees of freedom in DBG would lead to topological excitations of quarter vortices. If so, in DBG but not in TMDs, there would be a prefactor of 1/16 in Equation (18). This would dramatically reduce $T_{B K T}$.

\section{Conclusions}

Table 4 collects the relevant properties of double layer systems that have been proposed in the search for electron-hole superfluidity. Of these systems, double TMD monolayers have been shown in this paper to be exceptional candidates.

Table 4. Double layer system properties relevant for electron-hole superfluidity.

\begin{tabular}{lcccc}
\hline Masses Ratio & $\begin{array}{c}\text { Effective } \\
\text { Masses }\end{array}$ & $\begin{array}{c}\text { Valence Band } \\
\text { Screening }\end{array}$ & $\begin{array}{c}\text { Multicomponent } \\
\text { Superfluidity }\end{array}$ \\
\hline DQW & $m_{h}^{*} \sim 10 m_{e}^{*}$ & $0.07-0.3$ & no & no \\
DMG & $m_{h}^{*}=m_{e}^{*}$ & 0 & yes & no superfluidity \\
DBG & $m_{h}^{*}=m_{e}^{*}$ & 0.04 & yes & no \\
TMD & $m_{h}^{*} \sim 1.3 m_{e}^{*}$ & $0.4-0.5$ & no & yes \\
\hline
\end{tabular}

Unlike in DMG, the parabolic bands in TMDs allow the system to enter the strongly interacting BEC regime. In contrast to GaAs DQWs, where the mass imbalance weakens the superfluid gap, the effective masses in TMDs are almost equal. Furthermore, the effective masses in TMDs are relatively large, resulting 
in a much wider range of densities for electron-hole superfluidity. Valence band screening, which has a substantial negative effect in DBG, is negligible in TMDs because of its large band gap. The presence of the second condensate in some of the TMD systems reinforces the superfluidity, and thus maximizes density ranges and transition temperatures.

Author Contributions: Conceptualization, S.C., D.N., F.M.P. and A.P.; methodology, S.C., D.N. and A.P.; software, S.C.; validation, S.C., D.N., F.M.P. and A.P.; formal analysis, S.C. and A.P.; investigation, S.C., D.N., F.M.P. and A.P.; resources, S.C.; data curation, S.C.; writing-original draft preparation, S.C., D.N. and A.P.; writing-review and editing, S.C., D.N., F.M.P. and A.P.; visualization, S.C.; supervision, D.N., F.M.P. and A.P.; project administration, D.N. and A.P.; funding acquisition, F.M.P. and A.P. All authors have read and agreed to the published version of the manuscript.

Funding: This work was partially supported by the Fonds Wetenschappelijk Onderzoek (FWO-Vl), the Methusalem Foundation and the FLAG-ERA project TRANS-2D-TMD.

Conflicts of Interest: The authors declare no conflict of interest.

\section{Abbreviations}

The following abbreviations are used in this manuscript:

2D Two-dimensional

DQW Double Quantum-Wells

DMG Double Monolayer Graphene

hBN hexagonal Boron Nitride

DBG Double Bilayer Graphene

TMD Transition Metal Dichalcogenides

BEC Bose-Einstein Condensation

BKT Berezinskii-Kosterlitz-Thouless

\section{References}

1. Zhu, X.; Littlewood, P.B.; Hybertsen, M.S.; Rice, T.M. Exciton Condensate in Semiconductor Quantum Well Structures. Phys. Rev. Lett. 1995, 74, 1633. doi:10.1103/PhysRevLett.74.1633. [CrossRef]

2. Croxall, A.F.; Das Gupta, K.; Nicoll, C.A.; Thangaraj, M.; Beere, H.E.; Farrer, I.; Ritchie, D.A.; Pepper, M. Anomalous Coulomb Drag in Electron-Hole Bilayers. Phys. Rev. Lett. 2008, 101, 246801. doi:10.1103/PhysRevLett.101.246801. [CrossRef] [PubMed]

3. Seamons, J.A.; Morath, C.P.; Reno, J.L.; Lilly, M.P. Coulomb Drag in the Exciton Regime in Electron-Hole Bilayers. Phys. Rev. Lett. 2009, 102, 026804. doi:10.1103/PhysRevLett.102.026804. [CrossRef] [PubMed]

4. Lozovik, Y.E.; Yudson, V.I. Feasibility of superfluidity of paired spatially separated electrons and holes. JETP Lett. 1975, 22, 274. (Pis'ma Zh. Eksp. Teor. Fiz. 22, 556 (1975)).

5. Saberi-Pouya, S.; Conti, S.; Perali, A.; Croxall, A.F.; Hamilton, A.R.; Peeters, F.M.; Neilson, D. Experimental conditions for observation of electron-hole superfluidity in GaAs heterostructures. arXiv 2019, arXiv:1910.06631.

6. Neilson, D.; Perali, A.; Hamilton, A.R. Excitonic superfluidity and screening in electron-hole bilayer systems. Phys. Rev. B 2014, 89, 060502. doi:10.1103/PhysRevB.89.060502. [CrossRef]

7. Pieri, P.; Neilson, D.; Strinati, G.C. Effects of density imbalance on the BCS-BEC crossover in semiconductor electron-hole bilayers. Phys. Rev. B 2007, 75, 113301. doi:10.1103/PhysRevB.75.113301. [CrossRef]

8. Novoselov, K.S.; Geim, A.K.; Morozov, S.V.; Jiang, D.; Zhang, Y.; Dubonos, S.V.; Grigorieva, I.V.; Firsov, A.A. Electric field effect in atomically thin carbon films. Science 2004, 306, 666. doi:10.1126/science.1102896. [CrossRef]

9. Britnell, L.; Gorbachev, R.V.; Jalil, R.; Belle, B.D.; Schedin, F.; Katsnelson, M.I.; Eaves, L.; Morozov, S.V.; Mayorov, A.S.; Peres, N.M.; et al. Electron tunneling through ultrathin Boron Nitride crystalline barriers. Nano Lett. 2012, 12, 1707. doi:10.1021/nl3002205. [CrossRef] 
10. Min, H.; Bistritzer, R.; Su, J.J.; MacDonald, A.H. Room-temperature superfluidity in graphene bilayers. Phys. Rev. B 2008, 78, 121401. doi:10.1103/PhysRevB.78.121401. [CrossRef]

11. Lozovik, Y.E.; Sokolik, A.A. Coherent phases and collective electron phenomena in graphene. J. Phys. Conf. Ser. 2008, 129, 012003. [CrossRef]

12. Gorbachev, R.V.; Geim, A.K.; Katsnelson, M.I.; Novoselov, K.S.; Tudorovskiy, T.; Grigorieva, I.V.; MacDonald, A.H.; Morozov, S.V.; Watanabe, K.; Taniguchi, T.; et al. Strong Coulomb drag and broken symmetry in double-layer graphene. Nat. Phys. 2012, 8, 896. doi:10.1038/nphys2441. [CrossRef]

13. Lozovik, Y.E.; Ogarkov, S.L.; Sokolik, A.A. Condensation of electron-hole pairs in a two-layer graphene system: Correlation effects. Phys. Rev. B 2012, 86, 045429. doi:10.1103/PhysRevB.86.045429. [CrossRef]

14. Perali, A.; Neilson, D.; Hamilton, A.R. High-Temperature Superfluidity in Double-Bilayer Graphene. Phys. Rev. Lett. 2013, 110, 146803. doi:10.1103/PhysRevLett.110.146803. [CrossRef]

15. Burg, G.W.; Prasad, N.; Kim, K.; Taniguchi, T.; Watanabe, K.; MacDonald, A.H.; Register, L.F.; Tutuc, E. Strongly Enhanced Tunneling at Total Charge Neutrality in Double-Bilayer Graphene-WSe 2 Heterostructures. Phys. Rev. Lett. 2018, 120, 177702. doi:10.1103/PhysRevLett.120.177702. [CrossRef] [PubMed]

16. Conti, S.; Perali, A.; Peeters, F.M.; Neilson, D. Multicomponent screening and superfluidity in gapped electron-hole double bilayer graphene with realistic bands. Phys. Rev. B 2019, 99, 144517. doi:10.1103/PhysRevB.99.144517. [CrossRef]

17. Zhang, Y.; Tang, T.T.; Girit, C.; Hao, Z.; Martin, M.C.; Zettl, A.; Crommie, M.F.; Shen, Y.R.; Wang, F. Direct observation of a widely tunable bandgap in bilayer graphene. Nature 2009, 459, 820. doi:10.1038/nature08105. [CrossRef]

18. Mak, K.F.; Lee, C.; Hone, J.; Shan, J.; Heinz, T.F. Atomically thin $\mathrm{MoS}_{2}$ : A new direct-gap semiconductor. Phys. Rev. Lett. 2010, 105, 136805. doi:10.1103/PhysRevLett.105.136805. [CrossRef]

19. Jiang, H. Electronic band structures of molybdenum and tungsten dichalcogenides by the GW approach. J. Phys. Chem. C 2012, 116, 7664. doi:10.1021/jp300079d. [CrossRef]

20. Fogler, M.M.; Butov, L.V.; Novoselov, K.S. High-temperature superfluidity with indirect excitons in van der Waals heterostructures. Nat. Commun. 2014, 5, 4555. doi:10.1038/ncomms5555. [CrossRef]

21. Conti, S.; der Donck, M.V.; Perali, A.; Peeters, F.M.; Neilson, D. A doping-dependent switch from one- to two-component superfluidity at temperature above $100 \mathrm{~K}$ in coupled electron-hole van der Waals heterostructures. arXiv 2019, arXiv:1909.03411.

22. Wang, Z.; Rhodes, D.A.; Watanabe, K.; Taniguchi, T.; Hone, J.C.; Shan, J.; Mak, K.F. Evidence of high-temperature exciton condensation in two-dimensional atomic double layers. Nature 2019, 574, 76. doi:10.1038/s41586-019-1591-7. [CrossRef] [PubMed]

23. Strinati, G. A Survey on the Crossover from BCS Superconductivity to Bose-Einstein Condensation. Phys. Essays 2000, 13, 427. [CrossRef]

24. Randeria, M.; Duan, J.M.; Shieh, L.Y. Superconductivity in a two-dimensional Fermi gas: Evolution from Cooper pairing to Bose condensation. Phys. Rev. B 1990, 41, 327. doi:10.1103/PhysRevB.41.327. [CrossRef] [PubMed]

25. Pistolesi, F.; Strinati, G.C. Evolution from BCS superconductivity to Bose condensation: Role of the parameter $k_{F}$ ऊ. Phys. Rev. B 1994, 49, 6356. doi:10.1103/PhysRevB.49.6356. [CrossRef] [PubMed]

26. Singh, G.; Jouan, A.; Herranz, G.; Scigaj, M.; Sánchez, F.; Benfatto, L.; Caprara, S.; Grilli, M.; Saiz, G.; Couëdo, F.; et al. Gap suppression at a Lifshitz transition in a multi-condensate superconductor Nat. Mater. 2019, $18,948$. doi:10.1038/s41563-019-0354-z. [CrossRef]

27. Kosterlitz, J.M.; Thouless, D.J. Ordering, metastability and phase transitions in two-dimensional systems. J. Phys. C: Solid State 1973, 6, 1181. doi:10.1088/0022-3719/6/7/010. [CrossRef]

28. Saberi-Pouya, S.; Zarenia, M.; Perali, A.; Vazifehshenas, T.; Peeters, F.M. High-temperature electron-hole superfluidity with strong anisotropic gaps in double phosphorene monolayers. Phys. Rev. B 2018, 97, 174503. doi:10.1103/PhysRevB.97.174503. [CrossRef] 
29. Conti, S.; Perali, A.; Peeters, F.M.; Neilson, D. Multicomponent electron-hole superfluidity and the BCS-BEC crossover in double bilayer graphene. Phys. Rev. Lett. 2017, 119, 257002. doi:10.1103/PhysRevLett.119.257002. [CrossRef]

30. Xiao, D.; Liu, G.B.; Feng, W.; Xu, X.; Yao, W. Coupled Spin and Valley Physics in Monolayers of MoS 2 and Other Group-VI Dichalcogenides. Phys. Rev. Lett. 2012, 108, 196802. doi:10.1103/PhysRevLett.108.196802. [CrossRef]

31. Zhu, Z.Y.; Cheng, Y.C.; Schwingenschlögl, U. Giant spin-orbit-induced spin splitting in two-dimensional transition-metal dichalcogenide semiconductors. Phys. Rev. B 2011, 84, 153402. doi:10.1103/PhysRevB.84.153402. [CrossRef]

32. Kośmider, K.; González, J.W.; Fernández-Rossier, J. Large spin splitting in the conduction band of transition metal dichalcogenide monolayers. Phys. Rev. B 2013, 88, 245436. doi:10.1103/PhysRevB.88.245436. [CrossRef]

33. Van der Donck, M.; Peeters, F.M. Interlayer excitons in transition metal dichalcogenide heterostructures. Phys. Rev. B 2018, 98, 115104. doi:10.1103/PhysRevB.98.115104. [CrossRef]

34. Kumar, P.; Chauhan, Y.S.; Agarwal, A.; Bhowmick, S. Thickness and Stacking Dependent Polarizability and Dielectric Constant of Graphene-Hexagonal Boron Nitride Composite Stacks. J. Phys. Chem. C 2016, 120, 17620. doi:10.1021/acs.jpcc.6b05805. [CrossRef]

35. Yu, F.F.; Ke, S.S.; Guan, S.S.; Deng, H.X.; Guo, Y.; Lü, H.F. Effects of Se substitution and transition metal doping on the electronic and magnetic properties of $\mathrm{MoS}_{x} \mathrm{Se}_{2-x} / \mathrm{h}-\mathrm{BN}$ heterostructure. Phys. Chem. Chem. Phys. 2019, 21, 20073. doi:10.1039/C9CP03580J. [CrossRef] [PubMed]

36. Gerber, I.C.; Marie, X. Dependence of band structure and exciton properties of encapsulated WSe 2 monolayers on the hBN-layer thickness. Phys. Rev. B 2018, 98, 245126. doi:10.1103/PhysRevB.98.245126. [CrossRef]

37. Shanenko, A.A.; Aguiar, J.A.; Vagov, A.; Croitoru, M.D.; Milošević, M.V. Atomically flat superconducting nanofilms: Multiband properties and mean-field theory. Supercond. Sci. Tech. 2015, 28, 054001. doi:10.1088/0953-2048/28/5/054001. [CrossRef]

38. Vargas-Paredes, A.A.; Shanenko, A.A.; Vagov, A.; Milošević, M.V.; Perali, A. Cross-band versus intra-band pairing in superconductors: Signatures and consequences of the interplay. arXiv 2019, arXiv:1906.06528.

39. Lozovik, Y.E.; Sokolik, A.A. Multi-band pairing of ultrarelativistic electrons and holes in graphene bilayer. Phys. Rev. A 2009, 374, 326. doi:10.1016/j.physleta.2009.10.045. [CrossRef]

40. Kochorbe, F.G.; Palistrant, M.E. Superconductivity in a two-band system with low carrier density. J. Exp. Theor. Phys. 1993, 77, 442.

41. Giorgini, S.; Pitaevskii, L.; Stringari, S. Condensate fraction and critical temperature of a trapped interacting Bose gas. Phys. Rev. A 1996, 54, 4633. doi:10.1103/PhysRevA.54.R4633. [CrossRef] [PubMed]

42. Salasnich, L.; Manini, N.; Parola, A. Condensate fraction of a Fermi gas in the BCS-BEC crossover. Phys. Rev. A 2005, 72, 023621. doi:10.1103/PhysRevA.72.023621. [CrossRef]

43. Benfatto, L.; Capone, M.; Caprara, S.; Castellani, C.; Di Castro, C. Multiple gaps and superfluid density from interband pairing in a four-band model of the iron oxypnictides. Phys. Rev. B 2008, 78, 140502. doi:10.1103/PhysRevB.78.140502. [CrossRef]

44. Botelho, S.S.; Sá de Melo, C.A.R. Vortex-Antivortex Lattice in Ultracold Fermionic Gases. Phys. Rev. Lett. 2006, 96, 040404. doi:10.1103/PhysRevLett.96.040404. [CrossRef] [PubMed]

(C) 2020 by the authors. Licensee MDPI, Basel, Switzerland. This article is an open access article distributed under the terms and conditions of the Creative Commons Attribution (CC BY) license (http:/ / creativecommons.org/licenses/by/4.0/). 